\title{
Verzeichnis der Kunstwerke
}

nach Standorten geordnet.

(Dic Zahlen hinter den Namen bedeuten die Sciten. Nur die im Handbuch erwähnten Kunstwerke sind hier aufgeführt.)

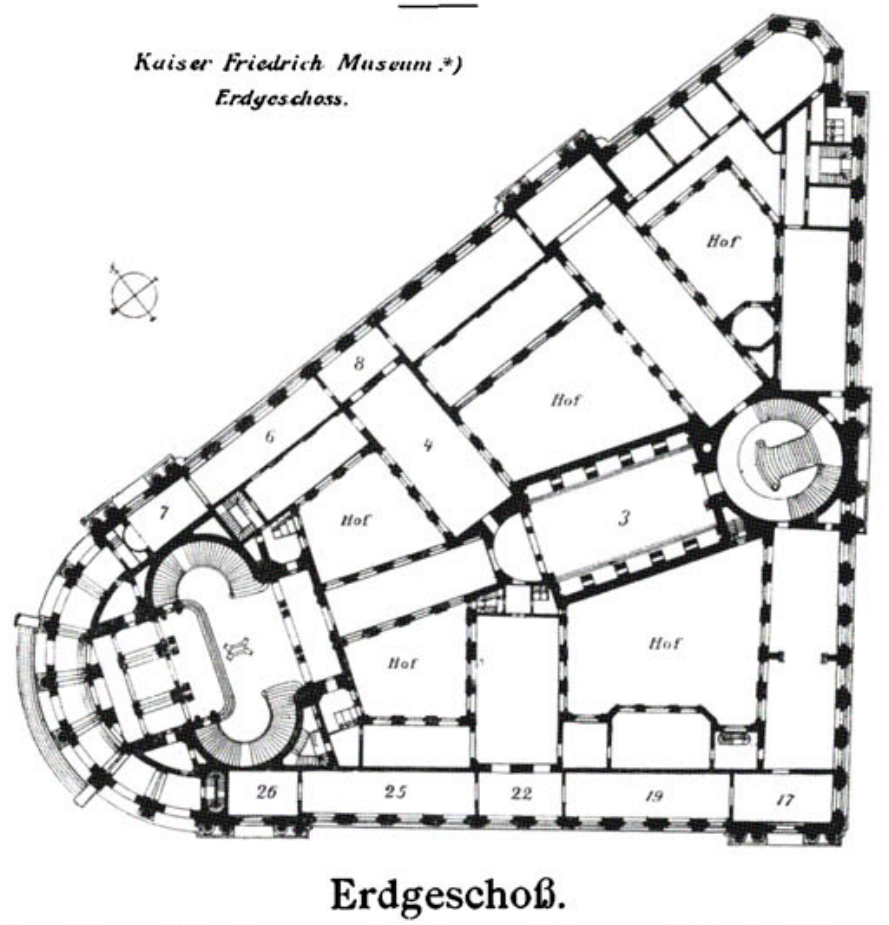

Saal 6 und 7. Frühchristliche und byzantinische Kunst.

Saal 7. Rechte Seitenwand. Rechte Gruppe.

Römisch. 4. Jahrhundert: Vier Apostel. Bruchstück von einem Sarkophag. Hochrelief. Marmor .........4.

*) Italienische Skulpturen befinden sich nur in den in obigem Plan mit Nummern versehenen Räumen. 
Römisch. 4. Jahrhundert: Das Opfer Isaaks. Bruchstück von einem Sarkophag. Hochrelief. Marmor .........

Römisch. 4. Jahrhundert: Sarkophag-Front mit unfertigem Doppelbildnis und S.-Kanneluren. Marmor.........

Linke Gruppe.

Römisch. 3. Jahrhundert: Zwei Sarkophagplatten mit Hirtenscenen. Hochrelief. Marmor ............. 3.

Römisch. 4. Jahrhundert: Sarkophag-Fragmente mit Darstellungen aus dem Hirtenleben u. a. ............

Römisch. 3.-4. Jahrhundert: Sarkophag mit schlafendem Jonas. Flachrelief. Marmor ............ 2, 3,

Saal 6. Rechte Seitenwand. Rechte Gruppe.

Byzantinisch. 5. Jahrhundert: Petrus als Zeuge eines Wunders. Marmorrelief aus Sinope ...............

Byzantinische Skulpturen des 4-6. Jahrhunderts ..... I4.

Linke Gruppe.

Byzantinisch. 10.-12. Jahrhundert: Marmorreliefs mit figürlichem und ornamentalem Schmuck ........... 4 .

Venezianisch. 12. und 13. Jahrhundert: Architektonische Zierstücke. Marmorreliefs mit symmetrischem Tierornament .........................

Longobardisch. 8.-9. Jahrhundert: Ciboriumsbogen mit Bandornament und Weihinschrift.............

8 u. 9. Venezianisch. 8.-9. Jahrhundert: Zwei Würfelkapitäle mit Kreuz und Akanthusornament (eins an der Fensterwand gegenüber aufgestellt) ...............

5. Longobardisch. 8. Jahrhundert: Ciboriumsbogen mit Bandornament in Flachrelief. Marmor.............

Presbyter Martinus (1 199): Maria mit dem Kind. Freigruppe. Holz. Bemalt und vergoldet ..............

2. Venezianisch. 9. Jahrhundert: Zweigeteiltes Fenster mit Monogramm Christi. Istrischer Kalkstein ......... 9.

Longobardisch. 8.-9. Jahrhundert: Viereckiger Brunnen mit ornamentalem Schmuck in Flachrelief ........... ro.

3 u. 4. Longobardisch. 8. Jahrhundert: Zwei Sarkophage mit Tier-, Bandwerk- und anderem Ornament in Flachrelief. Istrischer Kalkstein. (Der eine Sarkophag gegenüber am Fenster aufgestellt) ...............

Longobardisch. 8.-9. Jahrhundert: Runder Brunnen mit Bandornament. Kalkstein ...............

Schmalwand.

Lombardisch. Zwei Kapitäle mit Tiergruppen........ r4.

Venezianisch. 13. Jahrhundert: Archịtektonische Zierstücke mit Tier- und Pflanzenornament in Hochrelief ....... 14 . 
Oberitalienisch. I2. Jahrhundert: Steinbalken mit figurendurchsetzter Ranke .................

Unteritalienisch. I 2.-13. Jahrhundert: Simson bezwingt den Löwen und Simson schlägt die Philister. Zwei kleine Hochreliefs. Marmor ..................

Oberitalienisch. I2. Jahrhundert: Zwei lagernde Löwen als Säulenträger. Roter Sandstein ............. I 7 .

Oberitalienisch, um I200: Maria mit dem Kind. Statue. Stein, bemalt . . . . . . .

Toskanisch. 13. Jahrh.: Steinbalken mit stilisierter Ranke 20.

Erster Schaukasten (zunächst Saal 7).

Werkstatt des Florentius: Tonlampe mit Reliefdarstellung des guten Hirten .....................

Frühchristliche Lampen mit Christusmonogramm und anderem Reliefschmuck .................. 6.

Frühchristlich-römisch. 4. Jahrhundert: Diptychontafel mit Taufe Christi, Kindermord usw. Elfenbein. Flachrelief 7 .

2, 3. Ägyptisch. 4. Jahrhundert: Diptychon: Christus zwischen Petrus und Paulus und Maria zwischen zwei Engeln. Zwei Flachreliefs. Elfenbein $\ldots \ldots \ldots \ldots \ldots \ldots \ldots \ldots \ldots \ldots \ldots$.

Große Vitrine.

Altchristlich-römisch. 4. Jahrhundert: S. Petrus. Bronze-

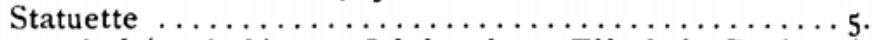

Oströmisch(-syrisch). 4. Jahrhundert: Elfenbein-Pyxis mit Darstellung des jugendlichen Christus und des Opfers Isaaks . . . .

Altchristliche Kleinkunst in Bronze und Elfenbein... 6, 7 .

Zweiter Schaukasten.

$67,68,69$. Unteritalienisch (salernitanisch?). 13. Jahrhundert: Drei Elfenbeinreliefs: Kreuzigung, Gefangennahme, Christus in Emmaus ...................

\section{Saal 8. Italienische Skulpturen des 13. und 14. Jahrhunderts.}

Eingangswand. Links.

Lombardisch. I 2. Jahrhundert: Basis eines Doppelsäulchens mit verschlungenem Tierornament ........... 7 . 28A. Römisch, r3. Jahrhundert(?): Bildnis eines Fürsten.

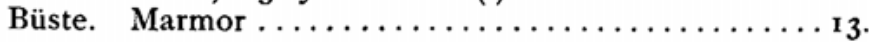

Rechts.

28. Süditalienisch, Ende des r3. Jahrhunderts: Bildnis einer jungen Fürstin. Büste. Marmor............ 
Oberitalienisch (Parma oder Modena). 13. Jahrhundert: Doppelkapitäl aus rotem Sandstein mit Darstellung der Monate . 17 .

Hauptwand. Rechte Gruppe.

22 A. Niccolo Pisano: Torso des Verkündigungsengels; von der Kanzel in Siena. Marmor ............... 27.

24. Giovanni Pisano: Lesepult mit Hochrelief, Christus zwischen zwei Engeln. Von der Kanzel in Pisa. Marmor 3I.

22. Niccolo Pisano: Lesepult mit Hochrelief: Bischof Buonacorso zwischen zwei Engeln. Marmor........... 27.

27. Toskanisch, Ende des r3. Jahrhunderts: Romanisches Kapitäl mit Masken- und Tierornament, in Hochrelief. Marmor ........................

31. Römisch, 14. Jahrhundert: Aschenurne. Marmor mit Dekoration in Mosaik ................... 3 .

55. Venezianisch, Ende des 14. Jahrhunderts: Eine weibliche Heilige(?). Statuette. Halbfigur. Marmor ...42. Mittelgruppe.

25. Andrea Pisano: Kruzifix. Rundfigur. Holz, bemalt 34 .

24. A-D. Nachfolger Giovanni Pisanos: Vier männliche Heilige. Statuetten. Marmor .............. Nachfolger Giovanni Pisanos: Zwei Engel. Statuetten. Marmor ............................ 23A. Giovanni Pisano: Zwei Sibyllen. Statuetten von der Kanzel in Pisa. Marmor ................ 26B. Pisanisch um $13^{80}$ : Verkündigung. Bemalte Holzfiguren, unter Lebensgröße ................ 23. Giovanni Pisano: Maria mit dem Kind. Statuette. Marmor ................

Linke Gruppe.

36. Venezianisch, Ende des r4. Jahrhunderts: Eine weibliche Heilige. Statuette. Halbfigur Marmor.....42.

Zweite Schmalwand.

29. 3o. Süditalienisch, 14. Jahrhundert: Leidtragender Mann und leidtragende Frau. Zwei Statuetten. Marmor ...38. 206. Sizilianisch um I 500: Maria mit dem Kinde. Statuette. Alabaster, vergoldet und teilweise bemalt ........

Saal 4. Spätgotische Bildwerke (provisorische Aufstellung).

Eing angsw and.

37. Venezianisch um r40o: Kruzifix. Rundfigur. Holz; bemalt . . . . . 
34 B. Giovanni d' Agostino: Maria mit dem Kind. Statue.

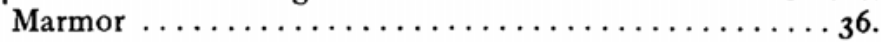

Linke Fensterwand.

r35. Robbia-Werkstatt, I6. Jahrhundert: S. Antonius der Einsiedler. Statue. Gebrannter Ton; bunt glasiert und bemalt ........................

In der Mitte.

162. Venezianisch. Erste Hälfte des I5. Jahrhunderts: Brunnenöffnung mit Hochreliefs. Istrischer Kalkstein . 42.

Fenster-Vitrine.

26. Nino Pisano: Maria mit dem Kind. Statuette. Alabaster; bemalt und vergoldet ...............

W and-Vitrine.

$24 \mathrm{H}$. Toskanisch um 1350: Maria mit dem Kinde. Statuette. Holz ..................

\section{Saal 26. Florentiner Tonbildner.}

Fensterwand.

107. Meister der Pellegrinikapelle: Maria mit dem Kind. Statuette. Gebrannter Ton; jetzt unbemalt.......6. 6 . 45. Donatello-Schüler: Maria mit dem Kind und musizierenden Engeln. Flachrelief. Stuck mit Resten von Bemalung ....................

Große Schmalwand. Oben.

rogD. Florentinisch zwischen 1400 und 1440: Maria mit dem Kind. Hochrelief. Stuck, bemalt ........64. ro8B. Florentinisch zwischen 1400 und 1430: Maria mit dem Kind in gotischem Tabernakel. Hochrelief. Gebrannter Ton, bemalt $\ldots \ldots \ldots \ldots \ldots \ldots \ldots \ldots \ldots 6_{3}$.

Unten.

32 A. Alberto di Arnoldo: Maria mit dem Kind. Flachrelief. Stuck, bemalt und vergoldet........... 151. 152. Art des Ghiberti: Verkündigung. Zwei Statuen. Holz, ursprünglich unbemalt ........... I $2 \mathrm{~A}$. Florentinisch $z$ wischen 1400 und 1425: Maria mit dem Kind über gotischem Laubwerk. Hochrelief. Gebrannter Ton; bemalt und vergoldet ........5 53, 63 .

Hauptwand. Oben.

rogC. Florentinisch zwischen 1400 und r440: Maria mit dem Kind. Hochrelief. Stuck, bemalt .........64. 
1x2B. Florentinisch zwischen 1400 und 1450: Maria mit dem schlafenden Kind. Hochrelief. Stuck, bemalt...63. ro8E. Florentinisch, erste Hälfte des Quattrocento: Maria mit dem Kind. Hochrelief. Gebrannter Ton, früher

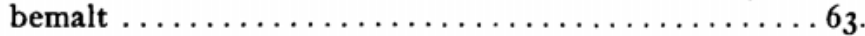

Unten.

ro9. Florentinisch zwischen 1420 und 1450: Maria mit dem Kind. Hochrelief. Stuck, bemalt .........6. 64 . ro8. Meister der Pellegrinikapelle: Maria mit dem Kind. Kleiner Altar. Gebrannter Ton, jetzt unbemalt ....63. $60 A$. Florentinisch um 1450: Maria mit dem Kind. Hochrelief. Stuck, bemalt ................. 108A. Florentinisch zwischen 1400 und 1450: Maria mit dem Kind. Kleiner Altar. Gebrannter Ton, bemalt und

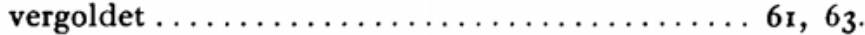

\section{Saal 25. Donatello und Luca della Robbia.}

Eingangswand. Links.

43. Donatello: Maria mit dem Kind. Flachrelief. Gebrannter Ton, unbemalt $\ldots \ldots \ldots \ldots \ldots \ldots \ldots \ldots \ldots \ldots \ldots \ldots$ I. 41. Donatello: Kreuzigung Christi. Flachrelief. Stuck, früher teilweise vergoldet $\ldots \ldots \ldots \ldots \ldots \ldots \ldots \ldots \ldots \ldots \ldots \ldots \ldots$.

Hauptwand.

Linke Gruppe. Unten.

I12D. Ghiberti: Maria mit dem Kind und Engeln in kleinem Tabernakelrahmen. Flachrelief mit Resten von Bemalung und teilweiser Vergoldung ..........62. 58A. Michelozzo: Maria mit dem Kind. Rundrelief. Stuck, bemalt und vergoldet $\ldots \ldots \ldots \ldots \ldots \ldots \ldots \ldots$ r.

46. Donatello: Maria mit dem Kind. Flachrelief in altem Tabernakel. Gebrannter Ton, unbemalt .......... 74 . 141. Florentinisch um 146o: Bildnis eines Ruccellai. Büste. Stuck, bemalt..................... 59. Agostino di Duccio: Maria mit dem Kind und Engeln. Flachrelief. Stuck, bemalt ........... 8. $_{3}$. ro6 H. Meister der unartigen Kinder: Caritas. Kleine Gruppe. Gebrannter Ton, jetzt unbemalt ........83. ro6B. Meister der unartigen Kinder: Maria mit dem Kind.

Kleine Gruppe. Gebrannter Ton, jetzt unbemalt....83. ro6E. Meister der unartigen Kinder: Maria mit dem Kind.

Statuette. Gebrannter Ton, bemalt .......... 83 . 47 u. 57 B. Donatello: Anbetung des Kindes. Zwei Halbreliefs. Stuck, bemalt $\ldots \ldots \ldots \ldots \ldots \ldots \ldots \ldots \ldots$ o. 
Mittelgruppe. Oben.

58. Michelozzo: Maria mit dem Kind. Hochrelief. Gebrannter Ton, bemalt und vergoldet $\ldots \ldots \ldots \ldots \ldots . \ldots$. 39 A. Donatello: Maria mit dem Kind und vier Cherubim. Hochrelief. Ton, bemalt und vergoldet. In altflorentinischem, ursprünglich nicht dazugehörigem Tabernakel . 74 . 49A. Donatello: Maria mit dem Kind. Flachrelief in altem Tabernakel. Stuck, bemalt und vergoldet ....8

Unten.

126. Francesco di Simone: Kamin mit Reliefs. Grüner Sandstein ...................

148. Florentinisch um r 490 : Bildnis des Lorenzo de' Medici. Büste. Stuck, bemalt . ........... 56, I 6 . 147. Florentinisch um 1490: Männliches Bildnis (sogen. Macchiavelli). Büste. Stuck, bemalt .......... $5^{6}$.

Rechte Gruppe.

1 6L. Luca della Robbia: Maria mit dem Kind. Hochrelief. Gebrannter Ton, bemalt und z. T. vergoldet. . go. I 5 . Luca della Robbia: Maria mit dem Kind und Engeln. Hochrelief. Stuck, bronzefarben bemalt .........89.

1 13. Luca della Robbia: Maria mit dem Kind und Engeln. Lünette aus Casa Alessandri. Gebrannter Ton, un-

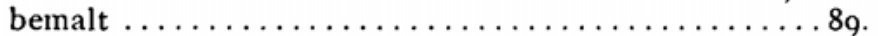

I 14 . Luca della Robbia: Maria mit dem Kind, mit Engeln und Heiligen. Halbrelief. Stuck, dunkel bronziert 54, 89.

I 6Q. Luca della Robbia: Maria mit dem Kind und Engeln. Flachrelief. Leder .............

Ausgangswand. Links.

I 7 . Luca della Robbia: Maria mit dem Kind. Hochrelief. Stuck, bemalt ....................

157. Luca della Robbia: Pietà. Halbrelief. Stuck, be-

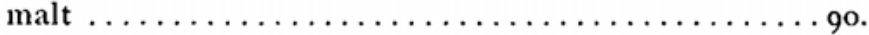

Rechts.

I 6. Luca della Robbia: Maria mit dem Kind. Hochrelief. Stuck, bemalt ................

Vitrine.

94. Verrocchio: Maria Magdalena. Statuette. Gebrannter Ton, bemalt und vergoldet .............. 26 . $589 \mathrm{D}$. Benedetto da Majano: Mariä Himmelfahrt. Kleines Hochrelief. Modell. Gebrannter Ton, unbemalt ...5 $5^{2}$. 106D. Meister der unartigen Kinder: Zwei sich balgende Kinder. Kleine Gruppe. Gebrannter Ton, bronziert .83. 
264 B. Giovanni da Bologna: Sieg der Tugend. Modell. Gebrannter Ton, unbemalt ................ 196. 27r A. Lorenzo Bernini: Modell eines Brunnens. Zwei Tritonen um einen Fisch kämpfend. Kleine Gruppe. Gebrannter Ton, unbemalt $\ldots \ldots \ldots \ldots \ldots \ldots \ldots \ldots 2$.

- Susini: Kardinal Medici. Tonstatuette. Modell zu der Marmorstatue in den Uffizien ................. 196. $638 \mathrm{~J}$. Giovanni da Bologna: Neptun und Triton. Kleine Gruppe. Tonmodell, vielleicht zum Brunnen am Palazzo

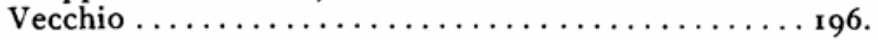

\section{Saal 22. Verrocchio.}

Eingangswand. Links.

62 F. Desiderio da Settignano: Maria mit dem Kind. Flachrelief. Stuck, bemalt. Komposition des Marmorreliefs bei

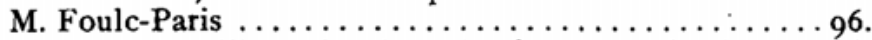

66. Ant. Rossellino: Maria mit dem schlafenden Kind. Halbrelief. Gebrannter Ton, bemalt und vergoldet . ro4.

Rechts.

62 B. Desiderio da Settignano: Maria mit dem Kind. Flachrelief. Stuck, bemalt und vergoldet. Komposition des Marmorreliefs in Turin, Pinakothek $\ldots \ldots \ldots \ldots \ldots \ldots 96$.

Hauptwand. Mitte.

62 D. Desiderio da Settignano: Christusknabe. Statuette. Stuck, bemalt ......................... 10o. A. del Verrocchio: Bildnis eines Jünglings. Büste. Gebrannter Ton, unbemalt ................. 26.

97 A. A. del Verrocchio: Grablegung Christi. Flachrelief. Gebrannter Ton, unbemalt $\ldots \ldots \ldots \ldots \ldots \ldots \ldots 5_{2}, \mathbf{1} 26$. 96. 97. A. del Verrocchio (Werkstatt): Zwei liegende, nackte Knaben. Statuetten. Gebrannter Ton, unbemalt ... 126.

Rechts.

93. A. del Verrocchio: Schlafender Jüngling. Statuette. Gebannter Ton, unbemalt ................

A usgangswand. Links.

I5oF. Matteo Civitale: Tabernakel mit dem Tod der Maria. Halbrelief. Tonmodell, nnvollendet und unbemalt . I19.

Fensterwand. Mitte.

63. Schule des Desiderio da Settignano: Maria mit dem Kind. Flachrelief. Stuck, früher bemalt .......... 54 . 


\section{Saal 19. Florentinische und oberitalienische Bildwerke der Frührenaissance.}

Eing angsw and.

Desiderio da Settignano: Maria mit dem Kind. Flachrelief. Stuck, bemalt und vergoldet. Komposition des florentiner Tabernakels in Via Cavour................ 96.

Hauptwand. Linke Gruppe.

87. Ben. da Majano: Vision Innozenz' III. Modell. Hochrelief. Gebrannter Ton, unbemalt ............ ro7.

72. Art des A. Rossellino: Maria mit dem Kind. Flachrelief. Stuck, z. T. vergoldet............. 54 . 149. Ben. da Majano: S. Katharina von Siena. Büste. Gebrannter Ton, bemalt und vergoldet......... r ro.

75. Ant. Rosellino: Maria mit dem Kind. Hochrelief ohne Grund. Gebrannter Ton, jetzt unbemalt ......... 64. Ant. Rossellino: Anbetung des Kindes. Hochrelief. Gebrannter Ton, unbemalt ........... 52, 106.

I49. Ant. Rossellino: S. Elisabeth. Büste. Gebrannter Ton, bemalt ....................

65. A. Rossellino: Maria mit dem Kind. Hochrelief ohne Grund. Gebrannter Ton, jetzt unbemalt ......... ro5. 92. Ben. da Majano: Kreuzigung Christi. Flachrelief. Stuck, bemalt und vergoldet $\ldots \ldots \ldots \ldots \ldots \ldots \ldots \ldots$ r 10.

85. Ben. da Majano: Bildnis des Filippo Strozzi. Büste. Gebrannter Ton, bemalt $\ldots \ldots \ldots \ldots \ldots \ldots \ldots \ldots 52$, 1 10.

Mittelgruppe.

I55A. Bart. Bellano: Maria mit dem Kind, Johannes und einem Engel. Flachrelief. Gebrannter Ton, mit Resten der teilweisen Vergoldung $\ldots \ldots \ldots \ldots \ldots \ldots \ldots$ r $35-136$. 19r D. Sperandio: Bildnis eines Bologneser Professors. Kolossalbüste. Gebrannter Ton, mit Resten von Be-

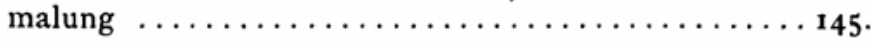

Rechte Gruppe. Oben.

191 A. Sperandio: Maria mit dem Kind und Cherubimen. Gruppe. Gebrannter Ton, bemalt ........... 445 . 163. Bart. Bellano(?): Johannes der Evangelist von Mönchen verehrt. Portallünette. Istrischer Kalkstein. Hochrelief $\ldots \ldots \ldots \ldots \ldots \ldots \ldots \ldots \ldots \ldots \ldots \ldots \ldots \ldots \ldots \ldots \ldots \ldots$ r 37.

In den beiden unteren Reihen.

19r C. Niccolo dell' Arca: Der heilige Bernhard. Statuette.

Stuck, bemalt ..................

156B. Paduanisch um 1480: Maria mit dem Kind. Hochrelief. Gebrannter Ton ............... 
191. Francia (?, Francesco Raibolini): Bildnis eines bolognesischen Edelmanns. Büste. Gebrannter Ton, bemalt I47. r66A. Bart. Bellano: Maria mit dem Kind. Hochrelief ohne Grund. Gebrannter Ton, früher bemalt...52, 135.

191 B. Bolognesisch um r450: Maria mit dem Kind. Statue. Gebrannter Ton, jetzt unbemalt ........... 4 47. 192B. Guido Mazzoni: Männliches Bildnis. Kopf. Gebrannter Ton, bemalt ................ 167. Art des Pietro Lombardi: Männliches Bildnis. Büste. Gebrannter Ton, unbemalt . . . . . .

- Ferraresisch, um r500: Trinität. Hochrelief. Gebrannter Ton, bemalt ...................

155. Domenico di Paris: Maria mit dem Kind. Hochrelief ohne Grund. Gebrannter Ton, früher bemalt . I43.

Ausgangswand.

199. Lombardisch um 1500: Begegnung des h. Dominikus und Franziskus. Halbrelief. Fichtenholz, bemalt und vergoldet ......................

Lombardische und venezianische Holzskulpturen .... I52.

Auf dem Tisch.

89D. Ben. da Majano: Maria mit dem schlafenden Kind. Statuette. Gebrannter Ton, bemalt ............

Saal 17. Italienische Bildwerke der Hochrenaissance. Eingangswand.

217. Pierino da Vinci: Die heilige Familie. Flachrelief. Stuck; bemalt.................... 218. Baccio Bandinelli: Selbstbildnis. Halbrelief. Gebrannter Ton, unbemalt ................

27 r C. Lorenzo Bernini: Dekorativer Entwurf für das Chorfenster von S. Peter in Rom. Tonmodell .........202.

Hauptwand links.

231. Jacopo Sansovino: Thronende Madonna mit Heiligen. Hochrelief. Gebrannter Ton, weiß bemalt und vergol-

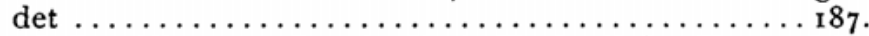

217 B-D. Vincenzo Danti: Drei Flachreliefs. Tonmodelle für die Basis der Hinrichtung Johannes d. T. am Battistero zu Florenz. (Eins hängt an der Eingangswand.) .... I9 I.

Auf den Wandbrettern und dem Tisch.

Raffaello da Montelupo: Johannes der Evangelist. Statuette. Tonmodell .........................

Tonmodelle von Giovanni da Bologna, Jacopo Sansovino und andern ................. 
Rechts.

249. Alessandro Vittoria: Bildnis des Admirals Contarini. Büste. Gebrannter Ton, unbemalt .......... 88. 265. Pietro Francavilla: Christuskopf. Flachrelief. Marmor. ...................... 232. Jacopo Sansovino: Maria mit dem Kind. Hochrelief. Gebrannter Ton, früher bemalt......... 86 .

Fensterwand.

225. Cristofero Romano (?): Bildnis der Teodorina Cibò. Büste. Marmor ... $\ldots \ldots \ldots \ldots \ldots \ldots \ldots \ldots \ldots$ i 84. 227. Art des Andrea Sansovino: Sturz des Phaeton. Hochrelief. Marmor .................

In der Mitte des Saales.

86. Benedetto da Majano: Maria mit dem Kind. Statue. Gebrannter Ton, bemalt ..............

\section{Saal 3. Basilika.}

Linke Seitenwand. Zweite Nische.

208A. Giovanni da Bissone: Die Hoffnung. Flachrelief.

Marmor ...................

Dritte Nische.

I 8 . Andrea della Robbia: Altar der Madonna zwischen vier Heiligen, aus Varramista. Gebrannter Ton, bunt glasiert.

.93.

Fünfte Nische.

128 A. Giovanni della Robbia; Beweinung Christi. Altargruppe. Gebrannter Ton, bemalt ...........

Hintere Schmalwand.

164. Bartolomeo Buon (?): S. Hieronymus. Hochrelief. Kalkstein ....................... 165, 166. Antonio u. Tullio Lombardi: Zwei Wappenhalter. Statuen. Marmor ................. 5 . 205. Römisch um 1500 : Bildnis Alexanders VI. (?) Kolossalbüste. Marmor .................... 197 A. Veronesisch, um 1490. Reitender Kardinal. Hochrelief ohne Grund. Marmor..............

Rechte Seitenwand. Fünfte Nische.

246. Alessandro Vittoria: Selbstbildnis. Büste. Marmor. (Ưber einem venezianischen Sarkophag von c. 1540.) I 88. 
Dritte $\mathrm{Nische}$.

I29. Robbia-Werkstatt, 16. Jahrhundert: Maria mit dem

Kind, Hieronymus und Johannes dem Täufer. Hochrelief. Gebrannter Ton, glasiert ............

Zweite Nische.

203. Luigi Capponi: Front eines Tabernakels. Hochrelief. Marmor ...................

Erste Nische.

259. Ant. Begarelli: Kreuzigung Christi. Altargruppe. Gebrannter Ton, marmorartig bemalt und vergoldet . 185.

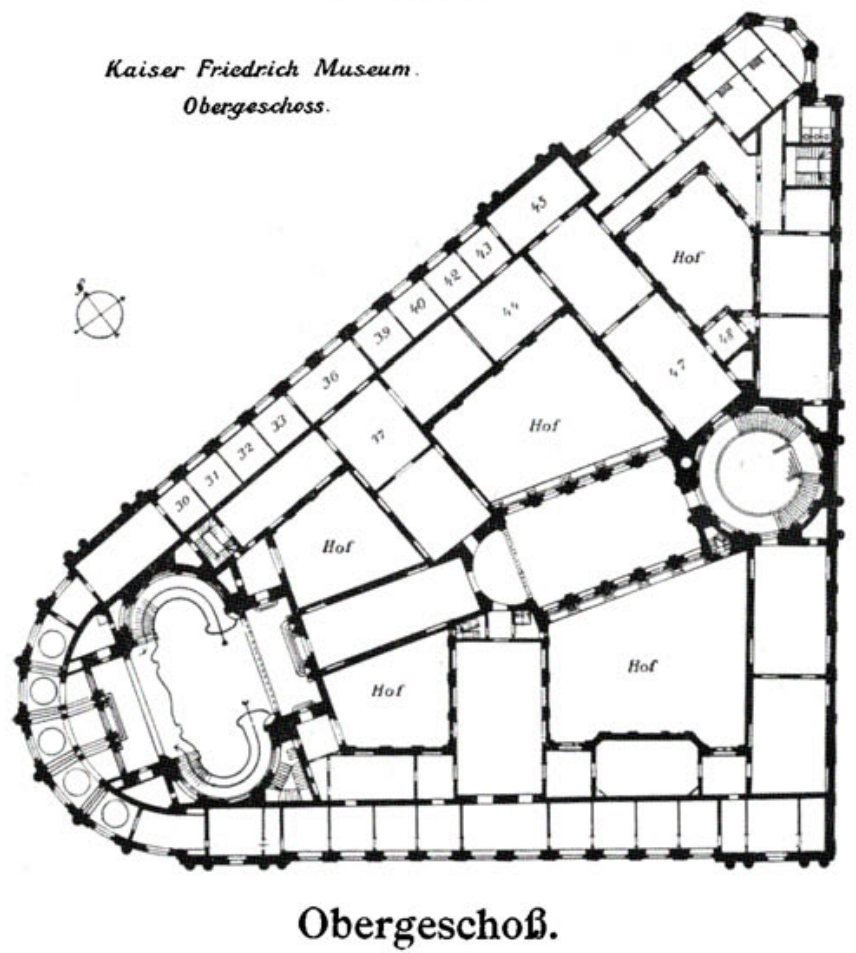

Kabinett 30. Kleinere Florentiner Gemälde des 15. Jahrhunderts.

Rückwand.

Benedetto da Majano: Tür mit Intarsiaschmuck ...... III. 
1 19. Andrea della Robbia: Maria mit dem Kind und zwei Engeln. Lünette. Hochrelief. Gebrannter Ton, glasiert und vergoldet $\ldots \ldots \ldots \ldots \ldots \ldots \ldots \ldots \ldots \ldots \ldots$.

\section{Kabinett 31. Robbia-Werke.}

Eingangswand. Oben.

I37 A. Werkstatt der Robbia, Komposition des Verrocchio: Kopf eines Kriegers. Hochrelief. Gebrannter Ton, bunt glasiert ............... Unten.

r16 P. Luca della Robbia: Maria mit dem stehenden Kind. Hochrelief. Gebrannter Ton, glasiert.......89. I 6 N. Luca della Robbia: Maria mit dem Kind, aus Casa Frescobaldi. Hochrelief. Gebrannter Ton, glasiert . 85. I 6 R. Luca della Robbia: Maria mit dem Kind, das einen Apfel hält. Hochrelief ohne Grund. Gebrannter Ton, glasiert ....................

Rückwand. Rechts.

I 19 D. Luca della Robbia: Jünglingskopf. Hochrelief. Gebrannter Ton, bunt glasiert ................

Links.

121. Andrea della Robbia: Knabe als Brunnenfigur. Statuette. Gebrannter Ton, bunt glasiert ...........93.

A usgangswand.

1 26. Werkstatt des A. della Robbia: Maria mit dem Kind, Johannes und zwei Engeln. Halbrelief. Gebrannter Ton, bunt glasiert .....................

- Luca della Robbia: Madonna zwischen zwei Engeln. Lünette. Hochrelief. Gebrannter Ton, glasiert. (Neue Erwerbung.) .....................

137 B. Art des Giovanni della Robbia: Pietà. Halbrelief. Gebrannter Ton, bunt glasiert .............

116 A. Luca della Robbia: Maria mit dem Kind. Halbrelief. Gebrannter Ton, bunt glasiert .......... 89.

I 9 A. Andrea della Robbia: Verkündigung. Halbrelief. Gebrannter Ton, bunt glasiert . . . . . . .

\section{Kabinett 32. Marmorwerke von Donatello,} Desiderio und andern Florentinern.

Eingangswand.

44. Werkstatt des Donatello: Maria mit dem Kind. Flach-

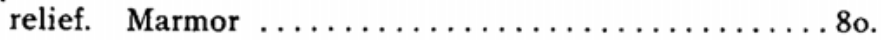


62. Desiderio da Settignano: Bildnis der Marietta Strozzi. Büste. Marmor .................. $98-99$. 39. Donatello: Madonna aus Casa Pazzi. Flachrelief.

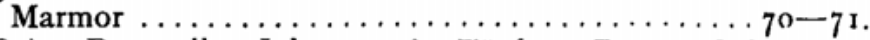
$3^{8} \mathrm{~A}$. Donatello: Johannes der Täufer. Büste. Gebrannter Ton, bemalt ......................

42. Donatello: Madonna aus Casa Orlandini. Flachrelief. Marmor ..........................

62 C. Desiderio da Settignano: Bildnis einer jungen Frau. Büste. Stuck, unbemalt . . . . . .

Rückw and:

- Art des Desiderio da Settignano: Eine Sibylle. Hochrelief. Marmor ..................... roo.

98, 99. Verrocchio: Bildnisse der Matthias Corvinus und Beatrice von Arragonien. Zwei Hochreliefs. Marmor 126. Verrocchio: Weibliches Bildnis. Flachrelief. Marmor . I 26. 37 B. Antonio di Banco (?): König David. Statue. Marmor ........................

Ausgangswand.

I 03 A. Werkstatt des Verrocchio: Zwei Putten mit ChristusMonogramm. Flachrelief. Marmor ........ 126-127.

84. Mino da Fiesole (?): Christus als Eccehomo. Büste. Marmor .......................

62 A. Desiderio da Settignano: Bildnis einer Prinzessin von Urbino. Büste. Kalkstein .......... 50, 99.

39 B. Donatello: Geißelung Christi. Flachrelief. Marmor 71 . 58 B. Andrea di Francesco Guardi (?): Maria mit dem Kind. Flachrelief. Marmor $\ldots \ldots \ldots \ldots \ldots \ldots \ldots \ldots \ldots \mathbf{8}_{3}$.

\section{Kabinett 33. Kleine Bronzewerke.}

In den Schaukästen der drei Wände sind Plaketten von Moderno, Andrea Riccio, Ulocrino, Caradosso (?), Sperandio, Giovanni Fiorentino (?), Valerio Belli und unbekannten, meist oberitalienischen Meistern ausgestellt; dazwischen auf Wandbrettern kleinere Reliefs und Statuetten 137-142.

Eingangswand.

443. Mailändisch um 1500: Begegnung des hl. Ambrosius mit Theodosius. Hochrelief. Bronze .......... 152.

Rü ckwand (auf und in der Libreria).

359, 360. Venezianisch: Petrus und Paulus. Zwei Statuetten. Bronze ....................... 5 57.

Benvenuto Cellini: Mythologische Darstellungen. Sechs Flachreliefs. Gold auf Lapis Lazuli-Grund ....... 192. 
Ausgangswand:

434. Antonio Lombardi: Segnender Christus mit der Weltkugel. Hochrelief. Bronze ............ 5 57.

Im Pultschrank, Fensterseite unten.

432. Ant. Filarete: Maria mit dem Kind und Fngeln.

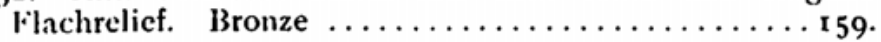

\section{Saal 36. Große und kleine Bronzewerke.}

Eingangswand.

Im ersten Schaukasten Statuetten (Nachbildungen antiker Kompositionen, Alessandro Vittoria: Die vier Fvangelisten Nr. 37 $1-374$ und andere Werke); in den andern Plaketten und kleine Reliefs von Donatello $(428$ Geißelung, 629, 630 Madonnen, 631-636 mythologische Darstellungen) und anderen Florentinern.

In der Mitte.

22. Gian Marco Cavalli (?) Bildnis des Giovanni Batista Spagnoli. Bronzebüste, reliefartig umrahmt ...... 2.36. J)onatello: Tilora oder Abundantia. Statuette. Bronze 74. 222. Antonio Rossellino: Bildnis eines Knaben. Büste. Bronze ........................... 234. I)onatello: Tamburinschlagender Engel. Vom Taufbrunnen in Siena. Statuette. Bronze ........ 70-71.

Längs wand.

220. Donatello: Bildnis Ludovicos III. Gonzaga. Büste. Bronze ....................... 78.

Schule von Bergamo um 1500: Zwei reichdekorierte Portale.

(I)ie Hauptansicht im Nebenraum Saal 37) ....... 152. 225. Italienisch um 1580: Bildnis Papst Gregors XIII. Büste. Bronze .....................

224. Florentinisch um 1550: Bildnis des Francesco del Nero. Büste. Bronze .................. 22 I. Oberitalienisch um r480: Bildnis Ludovicos III. Gonzaga. Büste. Bronze ................

Ausgangswand.

29r. Giovanni da Bologna: Nessus und Dejanira-Gruppe. Bronze ..................... Schaukästen mit Statuetten von Giovanni da Bologna, Candida und anderen .................

In der Mitte des Saales.

233. Donatello: Johannes der Täufer. Statuette. Bronze 69. Bode, Ital. Plastik. 
Erste Vitrine.

Bronzestatuetten von Ghiberti (232 Karyatide), Donatello (235 David, Art des D. 237 Amor), A. Pollaiulo (244 Paris, 245 Marsyas), Bertoldo (240 Hieronymus, 241 Herkules, 242, 243), Bellano (303 Hekate, 304-310) Riccio (5 I5 Wasserträger, 314 Schlafende Nymphe, 313, 316-321) und anderen Florentinern und Oberitalienern .. 141- 142 .

Zweite Vitrine.

Bronzestatuetten von Michelangelo (258 Schächer, 26o Simsongruppe nach Skizze M.s), Benvenuto Cellini und verwandten Künstlern $(26 \mathrm{I}-270)$, Giovanni da Bologna (290 usw.), T. und Ant. Lombardi, Aless. Vittoria und anderen $\ldots \ldots \ldots \ldots \ldots \ldots \ldots \ldots \ldots \ldots \ldots \ldots \ldots \ldots \ldots \ldots \ldots \ldots$.

\section{Kabinett 39. James Simon-Sammlung.}

Eingangswand.

24. Benedetto da Majano: Bildnis des Kardinals Raffaello Riario. Büste. Gebrannter Ton, unbemalt ........ rio. 38. Art des Francia: Bildnis eines Knaben. Büste. Gebrannter Ton mit Resten alter Bemalung ........ I47.

Rückwand.

25. Andrea della Robbia: Maria mit dem Kind und Engeln. Hochrelief. Gebrannter Ton, glasiert und vergoldet. Andere Arbeiten von Andrea della Robbia 93.

22. Nino Pisano: Maria mit dem Kind. Statuette. Mar-

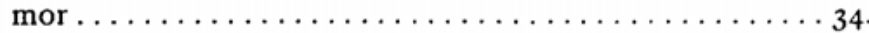

Ausgangswand.

23. Ben. da Majano: Maria mit dem Kind. Statuette. Gebrannter Ton, bemalt ................. rog.

In den Schaukästen dieser Wand und der Vitrine in der Mitte eine Sammlung von Medaillen von Vittore Pisano, Bertoldo u. a., Statuetten aus Bronze usw......... 14r.

Kabinett 40. Florentiner Marmorwerke der zweiten Hälfte des 15. Jahrhunderts.

Eingangswand. Oben.

81. Mino da Fiesole: Maria mit dem Kind. Flachrelief. Marmor . . . . . . . . .

77. Meister der Marmormadonnen: Maria mit dem Kind. Flachrelief. Marmor .................. 1 i6. 76. Meister der Marmormadonnen: Maria mit dem Kincl. Hochrelief. Marmor................ 


\section{Unten.}

79. Mino da Fiesole: Bildnis des Niccolo Strozzi. Büste. Marmor . . . . .

- Antonio Rossellino: Maria mit dem Kind. Flachrelief in altem Tabernakelrahmen. Stuck, bemalt....... I04. 79A. Mino da Fiesole. Bildnis des Alexo di Luca Mini. Büste. Marmor ..................

Rückwand.

154. Sienesisch um 1475: Maria mit dem Kind. Hochrelief. Marmor . . . . . . 153A. Federighi: Weibliches Bildnis. Büste. Marmor I 30. 62 E. Art des Desiderio da Settignano: Weibliches Bildnis. Büste. Stuck, bemalt und vergoldet ...........

82. Mino da Fiesole: Der Glaube. Flachrelief. Marmor. (Unvollendet.) . . . . . . . .

88. Ben. da Majano: Sockel einer Kirchenfahne. Marmor ...................

A usgangswand. Oben.

91 I). Werkstatt des Ben. da Majano: Zwei Putten mit dem Wappen der Gherardi. Lünette. Marmor........ II I. Unten.

8o. Mino da Fiesole: Bildnis eines jungen Mädchens. Büste. Marmor ................... 65 A. Ant. Rossellino: Maria mit dem Kind. Flachrelief. Marmor .................... 67. Ant. Rossellino: Bildnis eines jungen Mannes. Büste. Marmor .......................... ro6. $72 \mathrm{~A}$. Ant. Rossellino: Christus als Knabe. Büste. Sand-

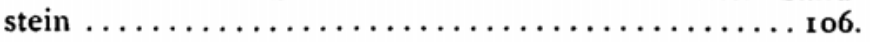

\section{Kabinett 42. Oberitalienische Skulptur und Malerei.}

Eing angswand.

183. Pietro Lombardi: Weibliches Bildnis. Flachrelief. Marmor . . . . . . . . .

6r. Francesco Laurana: Bildnis einer Prinzessin von Neapel. Büste. Marmor ...................

- Tamagnini: Bildnis des Salvago Acellini. Büste. Marmor ....................

153. Ant. Federighi (?): Bildnis eines Mannes. Flachrelief.

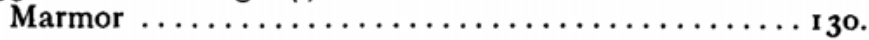

R ü ckwand.

208. Francesco Laurana: Weibliches Bildnis. Maske. Marmor . . . . . . . . . . 
20r B. Agost. Busti, gen. Bambaja: Die h. Anna selbdritt. Hochrelief. Marmor................

A usgangswand (über der Tür).

2or. Crist. Solari: Männliches Bildnis. Halbrelief. Marmor ...................

\section{Kabinett 43. Venezianische Bilder.}

Als Pfosten dienen an der Tür der Rückwand zwei Pilaster (Nr. 169, 170) mit Akanthus-Rankenornament, die aus der Werkstatt des Pietro Lombardi stammen ..... 50, I57.

\section{Saal 44. Venezianische Bilder.}

Eing angswand.

Genuesisch I5. Jahrhundert: Portal mit Reliefdarstellung der Georgs-Legende . ..................

Schmalwand. Mitte.

172. Meister von S. Trovaso: Altarvorsatz mit Fingeln. (Die mittleren halten Marterwerkzeuge, die äußeren musizieren.) Flachrelief. Marmor .............

\section{Saal 45. Italienische Bilder und Skulpturen des 16. Jahrhunderts.}

Links am Eingang.

209 A. Michelangelo Buonarroti: Apollo. Statuette. Marmor ...................

Schmalwand dem Eingang gegenüber. Oben. 23o A. Jacopo (Tatti) Sansovino: Maria mit dem Kind. Flachrelief. Papiermasse, bemalt und vergoldet.... 87 . 226. Andrea Sansovino: Bildnis des Kardinals Antonio del Monte. Hochrelief. Marmor ............. 180. 23o. Jacopo (Tatti) Sansovino: Maria mit dem Kind. Flachrelief. Papiermasse, bemalt ........... 877 .

Unten.

247. Alessandro Vittoria: Bildnis des Ottavio Grimani. Büste. Marmor .................... 209. Michelangelo Buonarroti: Johannes der Täufer als Jüngling. Statue. Marmor .........50, 170-171. 248. Alessandro Vittoria: Bildnis des Pietro Zeno. Büste. Marmor .................... 88. 
Saal 47. Italienische Werke des 17. und 18. Jahrhunderts.

Fing ang swand.

273. Francesco Maratti: Bildnis des Malers Carlo Maratti. Büste. Marmor ......................202.

- Aless. Algardi: Bildnis des Kardinals Landivio Zacchia. Büste. Marmor ...................

An der rechten Längswand.

27r. Domenico Guidi: Bildnis des h. Filippo Neri. Büste. Marmor ....................... 272 . Italienisch um $175^{\circ}$ : Bildnis des Papstes Benedikt XIV. Büste. Marmor .....................

Kabinett 48. Fresken des Tiepolo.

420. François Iuquesnoy, gen. Fiamingo: Cupido, den Bogen schnitzend. Statue. Marmor............ 202. 
